WellBeing International

WBI Studies Repository

$12-2008$

\title{
Reviewing Existing Knowledge Prior to Conducting Animal Studies
}

Andrew Knight

Animal Consultants International

Follow this and additional works at: https://www.wellbeingintlstudiesrepository.org/acwp_arte

Part of the Animal Experimentation and Research Commons, Animal Studies Commons, and the Bioethics and Medical Ethics Commons

\section{Recommended Citation}

Knight, A. (2008). Reviewing existing knowledge prior to conducting animal studies. ATLA-Alternatives to Laboratory Animals, 36(6), 709.

This material is brought to you for free and open access by WellBeing International. It has been accepted for inclusion by an authorized administrator of the WBI Studies Repository. For more information, please contact wbisr-info@wellbeingintl.org.

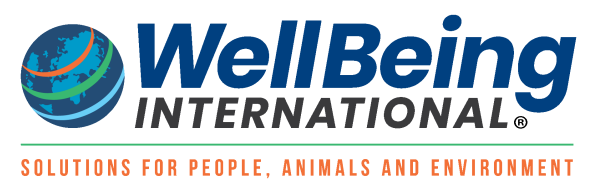




\title{
Comment
}

\section{Reviewing Existing Knowledge Prior to Conducting Animal Studies}

\author{
Andrew Knight \\ Animal Consultants International, London, UK
}

\begin{abstract}
Summary - Highly polarised viewpoints about animal experimentation have often prevented agreement. However, important common ground between advocates and opponents was demonstrated within a discussion forum hosted at www.research-methodology.org.uk in July-August 2008, by the independent charity, SABRE Research UK. Agreement existed that many animal studies have methodological flaws - such as inappropriate sample sizes, lack of randomised treatments, and unblinded outcome assessments - that may introduce bias and limit statistical validity. There was also agreement that systematic reviews of the human utility of animal models yield the highest quality of evidence, as their reliance on methodical and impartial methods to select significant numbers of animal studies for review, serves to minimise bias. Unfortunately, disagreement remained that animal experimental licence applications should reference systematic reviews of existing studies, before approval. The UK Medical Research Council requires that researchers planning human clinical trials must reference such reviews of related previous work. Existing knowledge is thereby fully and appropriately utilised, and redundant experimentation is avoided. However, objections were raised that a similar requirement would interfere with animal experimental licensing, because, to date, there have been very few systematic reviews of animal studies. In fact, the relative dearth of such reviews is a matter of considerable concern, and may partially explain the very poor human success rates of drugs that appear safe and/or efficacious in animal trials. Nevertheless, the disturbing number of human trials which have proceeded concurrently with, or prior to, animal studies, or have continued despite equivocal evidence of efficacy in animals, clearly demonstrate that many researchers fail to conduct adequate prior reviews of existing evidence. Where neither sufficient primary studies, nor systematic reviews of such studies, exist, for citation within a licence application, researchers should be able to provide evidence of this shortcoming, and, concurrently, demonstrate that the available literature and evidence have been adequately reviewed. This should also enable them to clearly demonstrate the need and scientific appropriateness of their proposed study, the validity of its design, and - importantly - that the benefits are reasonably likely to exceed the animal welfare, bioethical and financial costs. Invasive animal studies should never be permitted solely on the basis of less probable, speculative or intangible human benefits, or the mere satisfaction of scientific curiosity.
\end{abstract}

Key words: animal experiment, animal study, licence application, systematic review, Three Rs.

Address for correspondence: A. Knight, 91 Vanbrugh Court, Wincott Street, London SE11 4NR, UK. E-mail via: www.AnimalConsultants.org

\section{Introduction}

The high level of polarisation in the debate surrounding animal experimentation has often prevented agreement, and confused onlookers. It was therefore heartening to read the recent contribution to an online discussion forum by Simon Festing, Director of the Research Defence Society (RDS). It is clear that, despite our differences, those of us who are often critical of animal experiments and those who frequently support them, do share some important common ground.

The forum was hosted in July-August 2008, at http://www.research-methodology.org.uk, by the independent charity, SABRE Research UK.
SABRE aims to represent the interests of patients and research volunteers by calling for "rigorous scientific methods to assess the medical value of research involving animals", and avoids links with any political parties, animal lobby groups or the pharmaceutical industry (http:// www.sabre.org. uk). The discussion in question focused on the refusal of the RDS, the National Centre for the Replacement, Refinement and Reduction of Animals in Research (NC3Rs), and the Association of Medical Research Charities (AMRC), to sign a SABRE petition, available at http://petitions.number10.gov.uk/methodology/, stating that:

"We the undersigned petition the Prime Minister to improve methods to evaluate the validity and rele- 
vance of animal experiments by requiring that: (i) animal researchers are trained in experimental design and statistics; (ii) each licence application includes references to systematic reviews of existing relevant studies; (iii) approved applications are made available to researchers and others with relevant interest; and (iv) only the highest quality experimental designs of animal studies and laboratory practice are permitted."

On the face of it, such objectives appear eminently reasonable. It is well recognised that many animal studies employ inappropriate sample sizes. Excessive numbers may waste animal lives and other resources, whilst insufficient numbers may invalidate the experimental results obtained, leading to unnecessary repetition. The quality of the protocols used also varies considerably.

Suggested requirement (ii), for references to systematic reviews of existing relevant studies in project licence applications, is similar to the UK Medical Research Council (MRC) requirement that aims to ensure that researchers who are planning human clinical trials appropriately reference systematic reviews of related previous work, before proceeding (1). In this way, forthcoming experiments make the best use of existing knowledge, and redundant experimentation is avoided. Suggested requirement (iii) is similarly necessary, to guide concurrent or future research, prevent unnecessary experimental duplication, and permit the legitimate scrutiny of the merits of research.

It was surprising and disappointing that the RDS, AMRC and NC3Rs felt unable to add their names to this petition. This appears to represent a missed opportunity to help create a broad and united front in support of such laudable and obviously necessary measures. The NC3Rs declined to comment on their refusal to sign the petition.

\section{The AMRC Response}

In its comment, the AMRC stated that, "While the petition highlights the very real issue of ensuring that animal research is carried out to high standards, we feel its aims and desired outcomes are unclear and that it may amount in the end to little more than an awareness raising exercise."

In fact, the aims and desired outcomes of the petition were explicitly stated. In addition, it is surprising that the AMRC could consider increased awareness about best practice to be in any way problematic. The first step in achieving consistency of best practice within animal research is clearly the education of researchers, funding agencies, ethics committees and journal editors, concerning the importance of compliance with best practice principles. It is elementary that we should make the most of any opportunity to do so.

\section{The RDS Response}

The objections to this petition cited by the RDS were even more interesting. During their investigations, out of 19 current titles in the SABRE list of relevant publications, the RDS had apparently managed to randomly select all three of my own listed publications, within a sample size of four! Whilst I appreciate the attention afforded to my studies, the RDS then criticised the objectivity of my publications on the grounds that they considered me to be a "well-known antivivisectionist". No further data or reasons were provided. Although not relevant to the points raised within the SABRE petition, they cited these objections in support of their reasons for declining to sign the petition.

I am unsure what exactly the RDS meant by calling me a "well-known antivivisectionist". My main claim to such 'fame', if it may be so termed, was my involvement in establishing a very successful veterinary surgical training programme that involved neutering homeless cats and dogs to increase their chances of adoption, rather than killing healthy animals, when I was a veterinary student. Surely the RDS cannot disapprove of that?

Since then, I have published a series of critical assessments on the contributions of various animal models toward human biomedical advancement. To minimise bias, large numbers of published animal studies were selected randomly, or via other methodical and impartial means. For example, in some cases, all the studies within certain toxicity database subsets have been examined, without any discrimination. The results have been published following rigorous peer-review, including in ATLA (2), and neither the RDS nor any other party has published any contrary evidence.

These results clearly demonstrate that, while links to human healthcare advancements do exist, the utility of animal models for such purposes is generally poor. Simply stated, animal experimentation is not usually an efficient means of advancing human healthcare. These results are, I believe, highly repeatable, and not difficult to demonstrate, by anyone interested in critically assessing the value of large numbers of randomly-selected, published animal studies.

\section{Common Ground}

In commenting further, however, Simon Festing of the RDS indicated some important consistencies between the viewpoints of advocates of animal experimentation, and of those who frequently oppose animal experiments. We appear to agree, for example, that many animal studies have methodological flaws - such as inappropriate sam- 
ple sizes, lack of randomised treatments, and lack of blinded outcome assessments - that may introduce bias into the interpretation of the results, and limit the statistical validity of any conclusions drawn. Such deficiencies also occur within humanbased studies, although far less frequently. We appear to agree that systematic reviews represent the top of the evidence hierarchy, because their reliance on methodical and impartial methods for selecting studies for review is intended to minimise selection bias, thereby maximising the reliability of any conclusions reached.

We also agree that links between animal studies and human healthcare advancements do exist. Given the millions of animal studies conducted to date, this is hardly surprising. The question is, how common are those links? Or, more importantly, how efficient are animal models of humans, when used to test the toxicity or efficacy of putative pharmaceuticals, or the benefits and adverse effects of other clinical interventions? Are the public health-related benefits worth the animal welfare, ethical and financial costs inherent within animal experimentation? Is it reasonably possible that alternative research strategies might have proven more efficient, had the available funds been redirected toward them instead?

Whilst some such questions are destined to remain speculative, answers to others may be indicated by systematic reviews of the utility of animal experiments. My recent, published review of 27 other systematic reviews of the human clinical and toxicological utility of various animal experimental models, provides one example (2).

Fortunately, it also appears likely that we can agree on three of the four SABRE petition objectives, namely, suggested requirements (i), (iii) and (iv).

\section{Systematically Reviewing Existing Knowledge}

As voiced by Simon Festing, the remaining contentious requirement appears to be that: "(ii) each licence application includes references to systematic reviews of existing relevant studies." He voiced the concern that some project licences might not be granted, should references to systematic reviews become required, because, to date, there have been very few systematic reviews of animal studies.

Indeed, it is true that few such studies exist within the field of animal-based research. The dearth of such systematic reviews is, in fact, a matter of grave concern, and may partially explain the very poor human success rates of drugs that appear to be safe and/or efficacious in animal trials. In fact, only $8 \%$ of all drugs progressing to human trials after demonstration of safety in animal studies gain US Food and Drug Admin- istration licensing approval (3). For example, despite the existence of literature on the efficacy of more than 700 drugs in treating experimental models of stroke (4), only recombinant tissue plasminogen activator and aspirin have been convincingly demonstrated to be efficacious in human clinical trials of treatments for acute ischaemic stroke (5-7).

The systematic evaluation of all the available and appropriate animal-based evidence, as well as the outcomes of any other relevant preclinical studies or evaluations, would provide a more realistic assessment of the likely benefits, or shortcomings, of various putative pharmaceuticals, and would significantly decrease the number of such compounds that progress to fruitless clinical trials.

It is partly because of this kind of reason that the MRC requires researchers who are planning human-based trials to reference systematic reviews of related previous work before proceeding (1). To facilitate the detection of toxicity, and to provide insights into the factors which affect efficacy, such reviews should also include all relevant animal research (8).

The need for a similar review process prior to the performance of animal studies is aptly demonstrated by the disturbing number of cases in which clinical trials have proceeded concurrently with, or prior to, animal studies, or have continued despite equivocal evidence of efficacy in animals (2). Such cases clearly demonstrate that a significant number of animal researchers - and even some human-based researchers - do not conduct adequate reviews of existing evidence, before commencing further studies.

It is clear that a comprehensive and rigorous assessment of the available evidence should be a more strict requirement, before further studies are permitted to commence. Where neither sufficient primary studies, nor systematic reviews of such studies, exist, for the purposes of citation within a licence application, researchers should be required to demonstrate evidence that this is the case, and also, that the available literature and evidence have been adequately reviewed. In doing so, they should also be able to demonstrate the need for their proposed study, and should be able to appropriately position their experimental hypotheses within the established body of knowledge.

Simon Festing also points out that certain information relevant to such a review may not yet have achieved publication, for example, where results are very recent, and that the rigorous inclusion criteria required of systematic reviews may prevent the inclusion of such information in the results. There is no reason, however, why such additional, relevant information, cannot be included in the discussion section of a systematic review, where such material is of obvious relevance and importance. 


\section{Conclusions}

One key reason why the MRC requires researchers to reference, or, where necessary, conduct, systematic reviews of related previous work before proceeding with further human studies, is that the ethical and financial costs of human studies are high. Whilst different in some respects, the animal welfare, bioethical and financial costs of animal-based research are also frequently high. To these must be added the human costs, when potential cures are delayed, because researchers have consumed valuable time and resources in the pursuit of false trails. Therefore, it is entirely appropriate that animal researchers should be similarly required to present evidence that all reasonably available, relevant knowledge has been adequately reviewed, whether or not by the inclusion of published systematic reviews, before invasive animal studies are permitted to proceed.

Such a review should clearly demonstrate the need and scientific appropriateness of the proposed study, within the context of existing knowledge, the validity of its design, and - importantly - that the benefits are reasonably likely to exceed the animal welfare, bioethical and financial costs. Such studies should never be permitted solely on the basis of less probable, speculative or intangible human benefits, or the mere satisfaction of scientific curiosity.

\section{References}

1. Pound, P., Ebrahim, S., Sandercock, P., Bracken, M. \& Roberts, I. (2004). Where is the evidence that animal research benefits humans? British Medical Journal 328, 514-517.

2. Knight, A. (2007). Systematic reviews of animal experiments demonstrate poor human clinical and toxicological utility. ATLA 35, 641-659.

3. Pippin, J.J. (2008). "MAP” for improving drug testing: Mandatory Alternatives Petition urges FDA to require use of replacements to animal testing. Genetic Engineering and Biotechnology News, Vol. 28, No. 5. Available at: http://www.genengnews.com/articles/ chitem. aspx?aid=2384 (Accessed 05.12.08).

4. Macleod, M.R., O'Collins, T., Horky, L.L., Howells, D.W. \& Donnan, G.A. (2005). Systematic review and meta-analysis of the efficacy of melatonin in experimental stroke. Journal of Pineal Research 38, 35-41.

5. The National Institute of Neurological Disorders and Stroke rt-PA Stroke Study Group (1995). Tissue plasminogen activator for acute ischemic stroke. New England Journal of Medicine 333, 1581-1588.

6. Chinese Acute Stroke Trial (CAST) Collaborative Group (1997). Randomised placebo-controlled trial of early aspirin use in 20,000 patients with acute ischaemic stroke. Lancet 349, 1641-1649.

7. International Stroke Trial Collaborative Group (1997). The International Stroke Trial (IST): a randomised trial of aspirin, subcutaneous heparin, or both, or neither, among 19,435 patients with acute ischaemic stroke. Lancet 349, 1569-1581.

8. Perel, P., Roberts, I., Sena, E., Wheble, P., Briscoe, C., Sandercock, P., Macleod, M., Mignini, L.E., Jayaram, P. \& Khan, K.S. (2007). Comparison of treatment effects between animal experiments and clinical trials: systematic review. British Medical Journal 334, 197-200. 